\title{
ALASKA GEOLOGIC LEXICON REFERENCE DATA
}

\author{
Compiled by
}

J.A. LeVORSEN

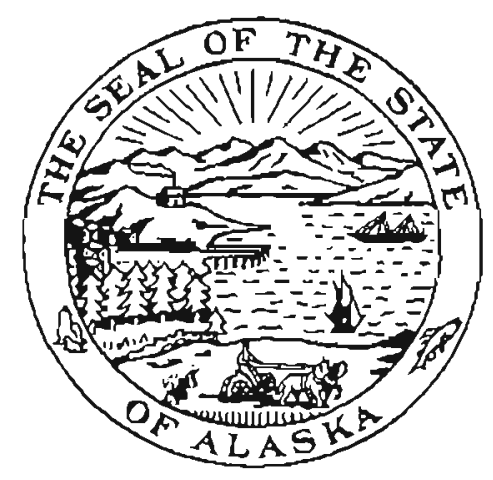

$M P-5$

Publishad by

DIVISION OF GEOLOGICAL ANO GEOPHYSICAL SURVEYS

ANCMORAGE, ALASKA

NOVEMEER 1973 


\title{
ALASKA GEOLOGIC LEXICON REFERENCE DATA
}

\author{
Compiled by \\ J.A. Levorsen \\ Petroleum Geologist \\ Division of Oil and Gas
}

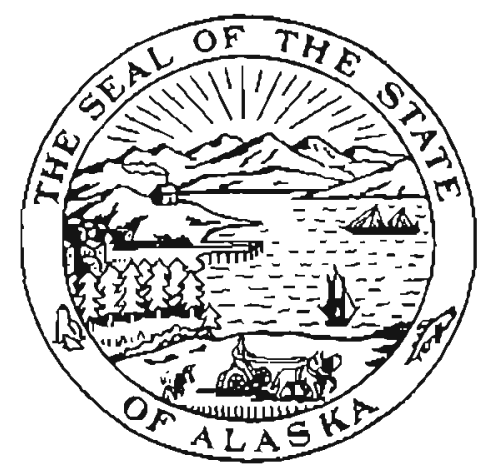

Publisnod by

DIVISION OF GEOLOGICAL AND GEOPHYSICAL SURVEYS

ANCMORAGE. ALASXA

NOVEMBER 1973 


\author{
STATE OF ALASKA \\ William A. Egan, Governor
}

\title{
DEPARTMENT OF NATURAL RESOURCES
}

Charles F. Herbert, Commissioner

\section{DIVISION OF GEOLOGICAL AND GEOPHYSICAL SURVEYS Donald C. Hartman, State Geologist}


FOREWORD

Geologists studying Alaskan stratigraphy are faced with increasing difficulty in trylng to keep abreast of current usage of stratigraphic names $1 \mathrm{n}$ the literature. Hopefully this index of lexicon reference data will prove helpful as a readily available listing of nomenclature currently in use. It should be of value between infrequent publication of geologic lexicons, as well as being the only listing of exclusively Alaskan names that also contains reference data.

The index provides an alphabetical listing of known stratigraphic names used in Alaska, annotated as to whether the names have been abandoned or rejected, or adopted for use by the U. S. Geological Survey. The system or serles to which the unit is assigned is given, as is the area in Alaska in whlch the name is applied. Additionally, a reference (listed at the end) is given to the lexicon entry, or to the entry in the annual "Changes in Stratigraphic Nomenclature" of the USGS, which cites the latest reference that affects usage of the name or the system/series assignment. Those names not appearing in these comprehensive publications have the appropriate reference cited.

Although the names Iisted have all appeared in generslly avallable geologic literature, they have not necessartly been formally deftned in accordance with the Code of the American Conmission on Stratigraphic Nomenclature. The index is intended as a reference ald for Alaskan stratigraphers, and not as an arbiter of stratigraphic nomenclature.

The data file from whlch this index is printed can be malntained current with relative ease, and we plan to update the index on an annual basis. The main problem will be the capture of pertinent data from the diverse geologic literature. The compiler will appreclate corrections or additions being brought to his attention, and most especlally, suggestions Eor Improvement.

The computer programing involved was a project of the compller. The computer cime required was made avallable through the courtesy of the Division of 011 and Gas. 
N.J ES:

1- DLASKAN STKATIGHAPHIC NAMES ANO DATA AS TAKEN FRUM THE U.S.G.5. LEXICONS FOK 1936-1967.

2. AODITIONAL NAMES FRDM LITERATURE AVAILABLE TO THE COMPILER, AND UNPUBL ISHEO SOURCES.

3. IDCATION FOR NAMES NOT IN THE LEXICCNS IS MERELY LISTED AS aLASKa: COMPILER HAS NOS

ANTICIPATED LEXICON AREA CLASSIFICATICA.

4. ABANOONED DR U.S.G.S. REJECTED NAMES IMDICATED GY +.

5. U.S.G.S. ADOPTFO NAMES INOICATEU BY *

6. PLFASE SEND CDRRECTIONS AND ADOIIIOHS TC THE COMPILER AT: ITEL. 279-14931, ALASKA OIVISION OF DIL AND GAS. 3CCI PERCUPINE ORIVE, ANCHORAGE, aLASKA 99501.

LOCATION

* ADAMS ARGILLITE

-admiralir islano volcanics

LCKER CAMBRIAN

AGATTU BEOS

ECCENE/OLIGOCENE

TERTIARY/CRETACEOUS

* AHTELL DIORITE

-axarura ilatiatich

AK ALURA ORIFT

* al apat limestone

IN LIS BUPNE GRDUP

*alapar mountain glactation

*al asktan glaciation

ALATNA GLACIATION

ALEUTS MEMBER OF KIALACVIK FORMAYION

CARBCNIFEROUS

$\begin{aligned} 1+2 & \text { CARBCNIFEROUS } \\ 11 & \text { PLEISTOCENE }\end{aligned}$

2

UPPER MISSISSIPPIAN

RECENT

RECENT

PLEISTOCENE

LOKER JURASSIC

*ambler glaciation

\#AMCHIIKA FORMATION

AMERICAN CREEK GRANITE

AMPHITHEATRE BASALY

AMY DOME METADIORITE, METAPERIOOTITE, HET AQASAL $T$

* anak tuVUK gruUPa

* anaktuvuk river glaciation

- aNAYAKNAURAK STADE

ANAYAKNAURAK SUBSTAGE, TILL, ORIFT

QANOREH GAY VOLCANICS

LEI STSCENE

LQHER TERTIARY

MESOZOIC

IRIASSIC

MIODLE OEVONIAN

LOKER CRETACEOUS

PLE I ST DCENE

PLEI STOCENE

TERT \AKY

* ANORFW LAKE FOPMATION
EAST-CENTKAL AL ASKA

SOUTHEASTERN AL ASKA

SOUTHHESTERN ALASKA

SOUTHEASTERN ALASKA

ALASKA

NORTHERN ALASKA

NORTHERN ALASKA

ALASKA

NORTHERN ALASKA

SOUTHWESTERN ALASKA

NORTHWESTERN ALASKA

SOUTHHESTERN ALASKA

HEST-CENTRAL ALASKA

SOUTHEASTERN ALASKA

EAST-CENTRAL ALASXA

NOEJ HERN AL ASKA

CENTHAL NORTHERN ALASKA

NURTHERN ALASKA

SOUTHHESTERN ALASKA

SOUTHWESTERN ALASKA 
*anivir lake stade

ANIVIK LAKE SUGSTAGE, DEIFT

*antLer valley STAOE aNtLER VALLEY SUBSTAGE, DRIFT

*arch poin't basalt

*arXose rioge formation

*ASHISHIK PASALT

ASSES EARS GRANTTE

-ayiyax member of seabfe formation

FBAIRO GROUP

* bandeo mountain stade EANDED MOUNTAIN SUBSTAGE, ORIFT

*aAnjo point formatigin

* BARLOH COVE FORKATION

THARRCh TRaIL MEMBER

OF SCHRADFR BLUFF FURMATION

* basin cRFex member

OF KATALLA FORMATION

- gay of pillars furmation

BEAR LAKE FTRMATION

BEAVER HAY GROUP

GELKOFSKI FORMATION

- beLKCFSKI TUFF

8ELUGA FORMATION

IN KENAI GROUP

* gergman Group

- bfrners formation

+HETTLES GRBUP OR SERIES

BIOUARKA FTEMAIION

- 8i gDFrakn afgill ite IN KANDIK GROUP

MBIRCH CRFEK SCHIST
RECENT

NORTHERN ALASKA

PLEI STOCENE

QUATERNAGY

CRETACEOUS

CENOZGIC

MESOZOIC

UPPER CRETACEOUS

PRE-MISSISSIPPIAN

PLE I STOCENE

E OCENE / OL I GOCENE

TRIASSIC/PERMIAN

UPPEA CRETACEOUS

CLIGLCENE

UPPER SLLURIAN

MI CCENE

LOHER TERTIARY

TERI I ARY

UPQER TERTIARY

TERTI ARY

CRET ACEOUS

JURASSICIPALEOZGIC

SILURIAN

LCWER JURASSIC

LUHER CRETACEOUS
NORTHERN ALASKA

ALASKA

SOUTH-CENTRAL ALASKA

SQUTHWESTERN ALASKA

WEST-CENTRAL ALASKA

NORTHERN ALASKA

NORTHERN ALASKA

NORT HERN ALASKA

SOUTHWESTERN ALASKA

SOUTHEASTERN ALASKA

NORTHERN ALASKA

SOUTHEASTERN AL ASKA

SOUTHEASTERN ALASKA

SOUTHWESTERN AL ASKA

SOUTHERN ALASKA

SOUTHWESTERN ALASKA

SOUTHHESTERN ALASKA

ALASKA

NORTMHESTERN ALASKA

SQUTHEASTERN ALASKA

NORT MERN ALASKA

SOUTHWESTERN ALASKA

EAST-CENTRAL ALASKA

EASTERN \& SOUTH-CENTRAL ALASKA 
HISHEP PEINT VOLCANIC MUDFLOH

* 8ITYER CREEK FORMATION

* black cap limestone

* black hills clactation

\$ BLACK POINT BASALT

BLACK RAPIDS ORIFT

BUKAN MOUNTAIN GRANITE

a Boutlegger cove Clay

BOULDER CPEEK QUAKTZ MONZONITE. GRANBD IORITE

- goundar granodLori I IN COAST RANGE INTRUSIVES

- bohser formation in tuxedon croup BOWSER MEMBER OF TUXEONI FORMATION

GRINE CREEK FORMATION

BRISTOL BAY SILTS AND GRAVELS

-brooks lake glaciation

BRDOKS RANGE GLACIATION

* brotrers vil. can ICS

IN STEPHENS PASSAGE GRDUP

*BROHNE GLACIATION

* buloir vol canjes

* burls creex shale member OF KAT ALLA FORMATION

* gurnt islano conglomerate IN HYO GROUP

* CALICD gLUfF Formation

* CAL IFORNIA CREEK MEMBER

OF TOTATL ANIKA SCFISY

*amille lava flom

* Cannery formatidon

\section{RECENT}

JURASSICJTRIASSIC

$1+2$

3

3

2

3

5

2

3

$1+2$

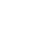

2

$1+2$

2

3

$\exists$

2

2

$\theta$

3

$1+2$
MIDDLE OEVONIAN

PLEISTOCENE

OUATERNARY

RECENT

JURASSIC/TRIASSIC

PLEI STOCENE

MESOZOIC

JURASSIC/CRETACEOUS

JURASSIC

CRETACEOUS

PLEISTOCENE

PLEI ST OCENE

NCY GIVEN

JURASSIC/CRETACEOUS

PLEISTOCENE

QUATERNARY

OLIGCCENE

UPPER TRIASSIC

UPPER MISSISSIPPIAN

MISSISSIPPIAN

PLEESTOCENE

LOKER PERMIAN

\section{ALASKA}

CanADA \& SOUTHEAST aLASKA

SOUTHEASTERN ALASKA

EASY-CENTRAL AL SKA

SOUTHHESTERN ALASKA

ALASKA

ALASKA

ALASKA

ALASKA

SOUTHEASTERN ALASKA

CENTRAL SOUTHERN ALASKA

NORYHERN ALASKA

SOUTHERN ALASKA

SOUTHEESTERN ALASKA

NORTHERN ALASKA

SOUTMEASTERN ALASKA

CENTRAL SOUTHERN ALASKA

SOUTHWESTERN AL ASKA

SOUTHEASTERN ALASKA

SOUTHEASTERN ALASKA

CENTRAL EASTERN ALASKA

ALASKA

ALASKA

SOUTHEASTERN ALASKA 
+ CAPE BEAUFURO COAL mEasuhes

* cape thompson member

OF NASORAK FORMATION

-CARIBOU HILLS GLACEATIDN

* Casadepaga schist

*cathedral valley agglomerate

*CEDar bay grantTe

* cedar cove formation

*CENOTAPH VOLCAMICS

CMAGVAN GL ACIATION

CHAGVAN ORIFT

* chamberl in glaciation

* chander formation IN NANUSHUK GROUP

* cmarior gRavel

CHEBANIKA STADE

※CHICKAL DON FORMATION

* CHIGNIK. FORMATION

* chinitina shale, siltstone. OR FM. CHINITNA SH. MBK. DIF SHELIKOF FH.

* chir ikgF formation

* chisik conglomerate mbr of naknex fim *CHISIK MBR OF NAXNEK FM

-chisna fijrmation

* CHIT IST ONF L IMESTUNE

* Chit itu formation

* ghitra point formation

CHUITNA MEMAER

CF TYONEX FORMATION

* Chunexsak fugmat ion

ECHUTE CREEK MEMBER

DF TGTATLANIKA SCHIST

\begin{tabular}{|c|c|}
\hline $1+2$ & CRETACEOUS \\
\hline 3 & LOWER MISSISSIPPIAN \\
\hline 2 & PLEISTOCENE \\
\hline $1+2$ & PRE-MISSISSIPPIAN \\
\hline 2 & UPPER TERTIARY \\
\hline $1+2$ & MESOZOIC \\
\hline 3 & OEVONIAN \\
\hline 8 & CLIGCCENE/MIOCENE \\
\hline 3 & PLEISTOCENE \\
\hline 3 & PLEISTOCENE \\
\hline 2 & CRETACEOUS \\
\hline 3 & PLETSTOCENE \\
\hline 3 & PLEISTOCENE \\
\hline$?$ & PALEOCENE \\
\hline $1+2$ & UPPER CRETACEOUS \\
\hline 8 & UPPER JURASSIC \\
\hline 12 & TERT IARY \\
\hline $1+2$ & UPPER JURASSIC \\
\hline $1+2$ & CARBONIFEROUS \\
\hline $1+2$ & UPPER TRIASSIC \\
\hline 10 & UPPER CRETACEOUS \\
\hline 10 & MICCENE \\
\hline 15 & TERT IARY \\
\hline 12 & MIOCENE \\
\hline$s$ & MISSISSIPPIAN \\
\hline
\end{tabular}

ALASKA

NORTHLESTERN AL ASKA

CENTRAL SOUTHERN ALASKA

NORYHWESTERN ALASKA

SOUTHHESTERN ALASKA

SOUTHEASTERN ALASKA

SOUTHEASTERN AL ASKA

SOUTH-CENTRAL ALASKA

SOUTHHESTERN ALASKA

AL ASK A

NORTHERN ALASKA

NORTHWESTERN ALASKA

NORTKERN ALASKA

CENTRAL SOUTHERN ALASKA

SOUTHWESTERN ALASKA

SOUTHWESTERN ALASKA

SOUTHWESTERN ALASKA

CENTRAL SOUTHERN ALASKA

SOUTHEASTERN ALASKA

EASTERN ALASKA

SOUTHERN ALASKA

SOUTHWESTERN ALASKA

ALASKA

SOU THEESTERN AL ASKA

ALASKA 
STRATIGRAPHIC NAME

* circle vol canics

- clamgulchian stage

CLARA CREEK GLACIATION

CLARA CREEK DRIFT

* clark peak sehist

CLEAR CREEK DRIFT

CLEAPY CREEX SERPENTINITE

COAL VALLEY MEMEER

OF CHIGNIK FORMATION

COAST RANGF DRORITE

acciast range intristues

*colville gaoup

COLVILLE SERIES

COIK INLET GRAVELS

COPPER MOUNTAIN GREENSTONE

OR AMPHIZOL ITE SCHIST

COPPER RIVER SILTS ANB GRAVELS

*CORNWALl IS LIMESTONE

IN HYD GRQUP

- coronados vol canics

- corw in furmation IN NANUSMUK GROUP

- crater crefk basalt

*CYNTRIA FALLS SS. IN IUXEDN! gRDUP CYNYHIA FALLS SS. MER.. TUXEONI FH

*DADINA SCHIST

* darl ing creek glaciation

*oelta glaciation

*oenal glaciation

aUESCON FGRMATION

* Donnelly glaciation
UPPER PALEOZOLC

MI CCENE/ PLI OCENE

PLEISTOCENE

$1+2$

3

3

3

$1+2$

$1+2$

$1+2$

$1+2$

$2+2$

$2+2$

3

11

$1+2$

2
LOCATION

EAST-CENYRAL ALASKA

SOUTH-CENTRAL ALASKA

SOUTHHESTERN ALASKA

SDUTHEASTERN ALASXA

ALASKA

EASY-CENTRAL ALASKA

SOUTHHESTERN ALASKA

ALASKA

CANADA E SOUTHEAST ALASKA

NORYHERN ALASKA

CENTRAL SOUTHERN ALASKA

SOUTHEASTERN ALASKA

SOUTMEASTERN ALASKA

SOUTKEASTERN ALASKA

SOUTHEASTERN ALASKA

NORT MWESTERN ALASKA

SOUTHWESTERN ALASKA

CENTRAL SOUTRERN ALASKA

SOUTHEASTERN ALASKA

EAST-CENTRAL ALASKA

EASY-CENTRAL ALASKA

CENTAAL ALASKA

SOUTHEASTERN ALASKA

EAST-CENTRAL ALASKA 
* gonble point oactie

*Douglas islano volcanic group

QOUGLAS ISLAND VBLCANICS IN STFPHENS PASSAGE GRQUP

* ory creek glaciation

*DUSHKIN GaSALT

- east capf volcanics

*FAST POINT FQRMATION

-ECHOOK a MEMBER

OF SADLFROCHIT FORMATION

+ECHODKA RIVER GLACIATION

* EDgecumbe vol canics

*EIDER PIINT Basalt

EIGHT LAKE GLACIATION EIGHT LAKE DRIFT

*EKLUTNA GL ACIATION

*EL I LIMESTDNE

IN BAIRD GROUP

- ENOICUTT GROUP

HENOCHKIN FORMATION

*esch creek glaciatiun

weshamy granite

+ESKA CONGL OMERATE

* estfer ash bec

*ESIHER GRANITE

-FAJRRANKS LOESS

* faneto furmayton

- fan mounta In GLacta trun

*FAREWELL CL.ASLIATION

HICKETT SERIFS

\begin{tabular}{|c|c|}
\hline 2 & QUATERNARY \\
\hline $1+2$ & JURASSIC/CRETACEOUS \\
\hline 3 & JURASSICICRETACEOUS \\
\hline 2 & PLEISTDCENE \\
\hline 2 & QUATERNARY \\
\hline 2 & GUATERNARY \\
\hline 2 & QUATERNARY \\
\hline 11 & UPPER PERMIAN \\
\hline 11 & PLEISTOCENE \\
\hline 3 & QUATERNARY \\
\hline 3 & CUATERNARY \\
\hline 3 & PLEISTOLENE \\
\hline 2 & PLEISTOCENE \\
\hline 3 & UPPER DEVONIAN \\
\hline 3 & MISSISSIPPIAN/DEVONIAN \\
\hline+2 & SURASIIC \\
\hline 3 & RECENT \\
\hline+2 & MESOZOIC \\
\hline+2 & MICCENE \\
\hline 2 & PLIISTOCENE \\
\hline+2 & PDLECZOIC \\
\hline 7 & PLEISTCCENE \\
\hline 12 & GUATERNARY/UPPER TERT IARY \\
\hline 2 & RECENT \\
\hline 2 & PLEISTOCENE \\
\hline & PALECLOIC \\
\hline
\end{tabular}

SOUTHWESTERN ALASKA SOUTHEASIERN AL ASKA SOUTHEASTERN ALASKA

CENYRAL SUUTHEKN ALASKA

SOUIHWESTERN ALASKA

SOUTHWESTERN AL ASKA

SOUTHWESIERN ALASKA

NORTHERN ALASKA

NORYHERN ALASKA

SDUTHEASTERN ALASKA

ALASKA

NDRTHERN ALASKA

ALASKA

NORT HWESTERN AL ASKA

NURTHESSTERN ALASKA

SOUTHWESTERN AL ASKA

WESTERIN ALASKA

SOUTHEASTERN ALASKA

CENTRAL SOUTHERN ALASKA

EAST-CENTRAL ALASKA

SOUTHEASTERN ALASKA

CENTRAL EASTERN ALASKA

SOUTHWESTERN ALASKA

NORTHERN ALASKA

CENTRAL SOUTHERN ALASKA

NORTHERN GENTRAL ALASKA 
* inger hay volcanics

* Fit I CREEK Siltstone IN TUXEONI GROUP

* EL AXMAN FORMATION

- DRo lake shale

FOREST CREEK FORMATION

* FortRESS mUUNTAIN FORMAIION

FURTYMILE GRANITE

tFTMEYMILE GROUP OR SERIES

* fossil creek volcanies

FIXX MILL FLOW

qFREOEYIXA FORMATION

FRESHHATEQ BAY FORMATION

- FROSTY PEAK VOL CANICS

* funNel CREEK limestone

* GaIXema sanostone IN tUXEDN I group ISS MBP OF KLALAGVIKITLXEONI FAS.I

GAKDNA DIORITE

* gakona furmation

* gambier bay formation

- gastineau vol canic group

* GEMISK GROUP

* cetmuna rmyol ite group

* GHOST ROCKS FORMATION

GLACIER BAY TILL OR FORMAIIIN

GLACIER RIDGE AGGLOMERATE

GLACIER RIDGE ANOESITE

* GlenN shale

* Gol DEN HiRn L IMESTONE LENTIL OF HASEN CREEK FM IN SXOLAI GRP

\begin{tabular}{|c|c|}
\hline 11 & LOWER TERTIARY \\
\hline 3 & MIDOLE JURASSIC \\
\hline $1+2$ & PLEISTOCENE \\
\hline 10 & MISSISSIPPIAN/OEVONIAN \\
\hline 3 & PLEISTOCENE \\
\hline 2 & IOHER CRETACEOUS \\
\hline $1+2$ & PRECAMBRIAN \\
\hline $1+2$ & PALECZOIC/PRECAMERIAN \\
\hline $1+2$ & HIDOLE ORDOVICIAN \\
\hline 3 & RECENT \\
\hline 11 & MICCENE \\
\hline 3 & UPPER DEVONIAN \\
\hline 3 & PLE ISTDCENE \\
\hline 3 & LOHER CAMBRIAN \\
\hline 4 & MIODLE JURASSIC \\
\hline 3 & MESOZOIC \\
\hline $1+2$ & EOCENE \\
\hline 3 & MIODLE DEVONIAN \\
\hline $1+2$ & UPPER JRTASSIC \\
\hline 2 & MESOZOIC/UPPER PALEOZOIC \\
\hline 2 & TERTIARY \\
\hline$\varsigma$ & PALEGCENE/EOCENE \\
\hline 3 & RECENT \\
\hline 2 & QUATERNARY \\
\hline 2 & QUATERnarY \\
\hline 10 & MESTZRIC \\
\hline 11 & LGKER PERMIAN \\
\hline
\end{tabular}

SOUT HHESTERN ALASKA

SOUTHEASTERN ALASKA

NOKTHERN ALASKA

EAST-CENTRAL ALASKA

SOUTHEASIERN ALASKA

NORTHERN ALASKA

EASTERN ALASKA

CENTRAL EASTERN ALASKA

NORTHEASTERN ALASKA

ALASKA

ALASKA

SOUTHEASTERN ALASKA

AL ASK A

EAST-CENTRAL ALASKA

CENTRAL SOUTHERN ALASKA

AL ASKA

SOUTHEASTERN ALASKA

SOUTHEASTERN ALASKA

SOUTHEASTERN ALASKA

SOUTHHESIERN ALASKA

SOUTHWESTERN ALASKA

SOUTHERN ALASKA

SOUTHEASTERN ALASKA

SOUTHHESTERN ALASKA

SOUTHWESTERN ALASKA

EAST-CENTRAL ALASKA 


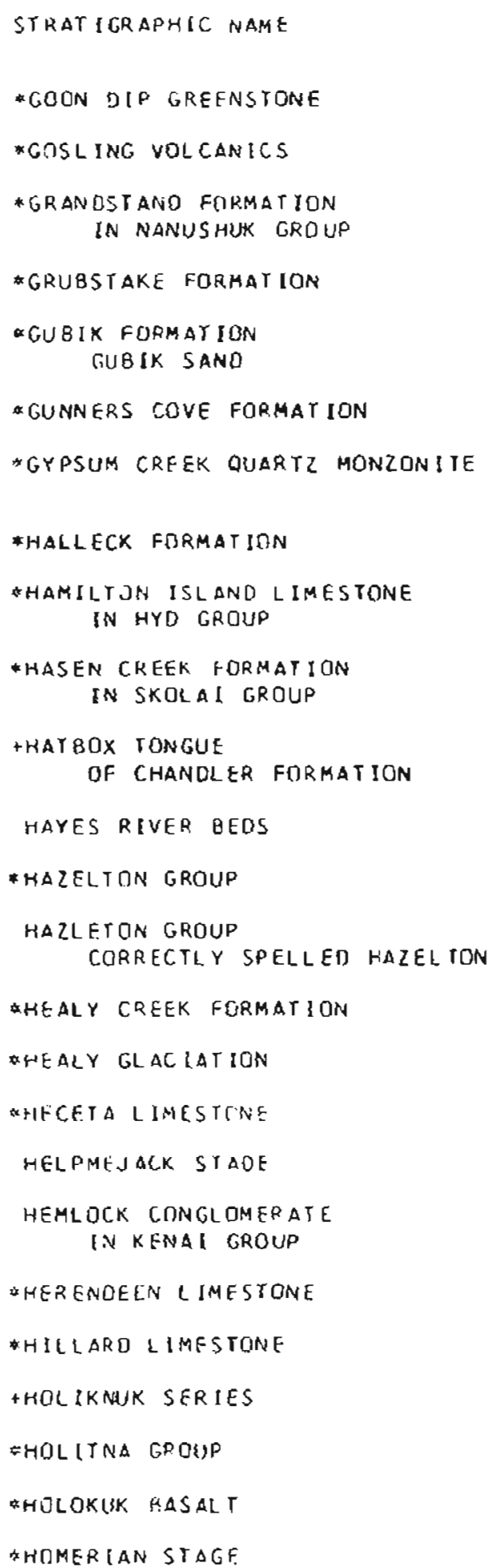

\section{SOUTHEASTERN ALASKA}

ALASKA

NORTHERN ALASKA

AL ASKA

NORYHERN ALASKA

SOUTHHESIERN ALASKA

SOUTHEASTERN ALASKA

SOUTHEASTERN ALASKA

SOUTHEASTERN ALASKA

ALASKA

NORTHERN ALASXA

SDUTHERN ALASKA

CaNaDa $E$ SOUTHEast alaska

CANAOA 2 SOUfHEAST ALASKA

ALASKA

CENTRAL, SOUTRERN ALASKO

SOUIHEASIERN ALASKA

NCRTHERN AL ASKA

AL $\triangle 5 K A$

SDUTHWESTERN ALASKA

EAST-CENTRAL AL ASKA

SOUTHERN AL ASKA

SOUTHWESTERN ALASKA

SOUTHWESTERN ALASKA

SOUTH-CENTRAL ALASKA 
4HOOD GAY FORMATION

HOODOO FORMATION

\#HORN MOUNT AIN IUFF MEMBER DF TALKEETNA FORMATION

- hound island vol canics IN HYD GRQUP

* HUNT FORK SHaLE IN ENORCOTT UROUP

*hurrat SLate

\# RYO FDRMAT ION OR GROUP

*hyder duartz monzonite

+ TOAK BASALT

*IOEFAROD BASALT

* IgNex formation

il yirak gravel

* imuruk vol canics

INIAKUK STADE

-iron caeek glaciation

*itkillik glaciatiun

ITKILYARIAK FORHATION IN ENDICOTT GROUP

* ivishak memger

OF SADLEFOCHIY FORMATION

qIYTUKEEN FORMATION

JACK LIMESTONE

JACKSINA FORMATION

JARW IS ASH BED

* Jatahmeind lake glaciation

* JOHNSTON hILL gLACIATION

* JONES HIDGE LIMESTINNE
DE VUNI AN

UPPER CREIACEOUS

LOMER JURASSIC

UPPER TRIASSIC

UPPER OEVONIAN

PRECAMBRIAN

$1+2$

2
SOUTHEASTERN ALASKA SOUTHHESTERN ALASKA SOUTHERN ALASKA

SOUTHEASTERN ALASXA

NCRTMERN ALASKA

NORTHWESTERN ALASKA SOUTHEASTERN ALASKA SOUTHEASTERN ALASKA

SOUTHWESTERN ALASKA SOUTHWESIERN ALASKA NORTHERN ALASKA

NORTHWESTERN ALASKA

ALASKA

NORTHERN ALASKA

WEST-CENTRAL ALASKA

CENTRAL NORTHERN ALASKA

ALASKA

NORTHERA ALASKA

SOUTHEASTERN ALASKA

SOUTH-CENTRAL ALASKA

SOUTHEASTERN ALASKA

EASTERN ALASKA

EAST-CENTRAL ALASKA

SOUTHWESTERN ALASKA

CANADA $\varepsilon$ EAST-CENTRAL aLASKa 
cjualin degatte

* KaguYAK FORMAT IOM

KAGUYAK FORMAIION

*kaltay formation

IN SHAKTOL IK GRCIUP

*kamishak foumarion KAMISHAK CHERT

A.KANAYUT CONGLOMERATE IN ENOLICOTT GROUP

* Kand IK gRoup

KANOIK FORMATION

KANEKTOK SILIS ANO GRAVELS

\#KANUT I GROUP

KAREN CREEK SANOSTONE

\#KARHEEN FDRMATION

*KaRLUK GLACIATION KARLUK ORIFT

+hasaan cafenstgne

*kitak glactation

*KATAKTURUK OOLOMITE

akatalla formatrgin

FKATHUL GKAYKACKE

IN KANDIK GROUP

EKASL I AN GROUP

HATMAI SERIES

FK $\triangle Y A K$ SHALE

IN FRDICOTT GaOUP

AKELNAN GUART $L$ ITE

IN KANOIK GRUUP

\#KEEVY PEAK FORMATION

-KTKKKTUK CONGLGMERATF IN FiNDICOTY GROUN

*KEKUJ VOLCANICS

\section{REFEREACE SYSTEH/SERIES}

$\begin{aligned} 1+2 & \text { WOHER CRETACEOUS } \\ 2 & \text { UPPER CRETACEOUS } \\ 2 & \text { UPPER CRETACEOUS } \\ 1+2 & \text { LORER CRETACEOUS } \\ 1+2 & \text { UPPER TREASSIC } \\ 3 \mathrm{C} & \text { UPPER DEVONIAN }\end{aligned}$

10

$1+2$

$1+2$

14

11

11

$1+2$

3
LOWEA CRETACEOUS

PLEISTOCENE

palegzoIC

UPPER TRIASSIC

DE VOA IAN/SI LUR IAN

PLEI STOCENE

LCHEA CRESACEOUS

RECENT

PRE-MESSISSIPPIAN

OLIGOCENE/MIOCENE

LOHER CREYACECUS

TRIASSICIPEKMLAN

JURASSIC

LCWER MISSISSIPPIAN

LCWER GRETACECUS

PALFCZOIC/ PRECAMBRIAN

MISSISSI PPIAN

UPPER TRIASSIC
LOCATION

SOUTHEASTERN AL SKA

CENTRAL SOUTHERN ALASKA

SOUTHERN ALASKA

CENTRAL WESTERN E CENTRAL ALASKA

CENTRAL SOUTHERN ALASKA

NORTHERN ALASKA

EAST-CENTRAL, ALASKA

CENTRAL SOUTHERN ALASKA

NORTHERN CENTRAL ALASKA

AL ASKA

SOUTHEASTERN ALASKA

ALASKA

SOUTHEASTERN ALASKA

AL ASKA

NORTHEASTERN ALASKA

SOUTHEASTERN ALASKA

EAST-CENTRAL AL ASKA

SOUTHEASTERN ALASKA

CENTRAL SOUTHERN ALASKA

NORFHERA ALASKA

FAST-CENTRAL ALASKA

ALASKA

NORTHEASTEKN ALASXA

SOUTHEASTERN AL SSKA 
*KELP GAY GROUP

KEMIK SANOSTONE MEMBER

OF OKPIKRUAK FORMATION

LEMUK GLACIATION KEMUX ORIFT

* Eenai formar Ion

KENAI GROUP

* ennel creek limestone

* Kennicott formation

* IALAGVIK formation

WKIELUAIK GROUP

*xilley stade

*KILI IK TUNGUF

OF CHANDLER FORMATION

- KINGAK SHALE

*KISIMHLOK FORMATION

aKISKa HARBOR FORMAT ION

*KL AHAK FORMAT ION

*KLUT INA gROLSP

* knik glaciation

* koeuk glaciation

* KOOIAK FORMATIIN

* kogosuk rlik tongue

OF PRINCE CREEK fORMATION

-XOGRUK FORMATION

IN LISBURNE GROUP

+KOLMAKOF SERIES

KOLOSH MEMBER

OF KIALAGVIK FORHATION

*KOOTZNAHOO FORMATYON

tKOTLO SERIES

* kotsina conclomerate

\begin{tabular}{|c|c|}
\hline 3 & JURASSICITRTASSIC \\
\hline 13 & LCHER CRETACEOUS \\
\hline 3 & PLEISTOCENE \\
\hline 7 & TERYIARY \\
\hline 16 & TERTIARY \\
\hline 3 & MIODLE DEVONIAN \\
\hline 10 & LOMER CRETACEOUS \\
\hline $1+2$ & MIDDLE JURASSIC \\
\hline 21 & PRECAMBRIAN \\
\hline 3 & PLEISTCCENE \\
\hline 2 & LGWER CREYACEOUS \\
\hline $1+2$ & JURASSIC \\
\hline 3 & LOHER CRETACEOUS \\
\hline 3 & QUATERNARY J UPPER TERT IARY \\
\hline 11 & PEANSYLVANIAN \\
\hline $1+2$ & PRE-PERMIAN \\
\hline 2 & $\rho_{L E I S T O C E N E}$ \\
\hline 3 & PLEISTOCENE \\
\hline$\S$ & CRETACEOUS \\
\hline 2 & UPPER CRETACEOUS \\
\hline 3 & MISSISSIPPIAN \\
\hline $1+2$ & TERYIARYIUPPER PALEOZOIC \\
\hline 2 & LOWER JURASSIC \\
\hline 3 & TERTIARY \\
\hline $1+2$ & PRE-MISSISSIPPIAN \\
\hline 7 & JURASSIC \\
\hline
\end{tabular}

SOUTREASTERN ALASKA

ALASKA

SOUTHHESTERN AL A SKA

CENYRAL SOUTHERN ALASKA

CENTRAL SNUTHERN ALASKA

SOUTHEASTERN ALASKA

SOUTHERN ALASKA

SOUTHKESTERN ALASKA

NORTHHESTERN AL ASKA

SOUTHERN ALASKA

NORTHERN AL ASKA

NORTHEASTERN ALASKA

NDRTHHESTERN AL ASKA

SOUTHERN ALASKA

SOUYHEASTERN ALASKA

SQUYHEASTERN ALASKA

ALASKA

NORTHMESIERN ALASKA

SDUTHERN ALASKA

NORTHERN AL ASKA

NORTHERN ALASKA

SOUTHERN ALASKA

SOUYHHESTERN ALASKA

SOUTHEASIERN ALASKA.

SIUTHEASTERN ALASKA

CENTRAL SOUTHERN ALASKA 
WKCUGAROK GRAVEL

+K DUGA AROK GRCUP

\#KCWAK ClaY

KOYUKUK GLACIASTON

* KEYUKUK GROUP

*keruglat gormatbon

TKUGRUK GROUP

- KugruK vol can IES

* kugururck format ton IN BAIRD GROUP

AKU IUS LIMESTONE

AKUKPEHRUK FORMATION IN NANUSHUK GROUP

*KULTHIETH FORMATION

KUPARUIK RIVER SANDS

* Kushtaka FORmat ION

HKUSKOKWIM GRAVELS AND SILTS

\#KUSKOKWIM GROUP

*Kuskulania formation

*Kuzitrin foRmation

* lavipones limesimae

H LAKF. GUART ZITF SCHIST

LANCSLIOE CKEFK GRANODIORITF

"LIGNITF CREEK FORMATION

* ISHURNE GROUP

LIS GUPNE L, IMESTONE

LITILE PAVLCF AGGLOMERATE

*L ETLE SITKIN Giacite

I. IVFNGDOD CMFRY

* Lost jIM lava flow
CENOZOIC

DEVONIAN/ ORDCVICI AN

$1+2$

$1+2$

3

$1+2$

12

$1+2$

12

3

3

2

12

18

$\theta$

$1+2$

2

$1+2$

$1+2$

11

$1+2$

3

$1 \mathrm{C}$

12

2

2

$1+2$

2
PLEISTGCENE

PLEISTOCENE

LOWER CRETACEOUS

IERTIARY/MESOZOIC

DE VONY AN/ ORDOVI I I AN

QUATERNARY/UPPER YERT IARY

UPPER DEVONIAN

UPPER SILURIAN

LCWER CRETACEOUS

LQHER YERTIARY

LOMER CRETACEOUS

EOCENE/OLIGOCENE

PLEISTDCENE

CRETACEOUS

UPPER IRIASSIC

DEVOKI AN

PENNSYLVANI AN

PRE-MISSISSIPPIAN

MESOZOIC

MICCENE

UPPER PALEOZOAC

QUATERNARY

QUATERNARY

MISSISSIPPIAN

GUATERNARY
ALASKA

NORTHWESTERN ALOSKA

NORTHWESTERN 2LASKA

NORTHEKN ALASKA

NORTH-CENTRAL ALASKA

SOUTHHESTERN ALASKA

NORYWHESTERN ALASKA

ALASKA

NORTMERN ALASKA

SOUTHEASTERN ALASKA

NORTHWESTERN ALASKA

SOUTHERN ALASKA

AL ASKA

SOUTH-CENTRAL E SOUTHEAST ALASKA CENYRAL SOUYHFRN ALASKA

SOUYHWESTERN ALASKA

ALASKA

NORTHWESTERN ALASKA

SOUYHEASTERN ALASKA

NORTHERN ALASKA

EASTERN ALASKa

OLASKA

NORYHERN ALASKA

SOUTHWESTERN ALASKA

SOUTHWESTERN AL ASKA

EAST-CENTRAL AL ASKA

CENTPAL WESTERN ALASKA 
MUIR TILL OR FORMATIOW

*MYSTIC CREER MEMRER

GF TUTATLANIKA SCHIST

- MCCANN HILL GHERT

+mCCARTHY cREek SHale

WMCEARTHY SHALE OR FORMATION

- maccoll ridge formation

MCHUGH COMPLEX

*naBesna limestone

WNAKNEK FORMATION

NAKNEX LAKE GRANITE

* NAKir ASINA GROUP

F NANOOK LIMESTONE

*NANUSHUK CROUP

NANUSHUK FORMATION

*Naptijhne glaciation

- NARROH CAPE FORMATIDN

WMASORAK FORMATION

IN I IS QUSNE GROUP

*NATION RIVER fIJRMATICN

*NFLCHINA L IME\$TINE

*NELLIE JUAN GRANITE

anTNANA GLAVEL

FNEPUCKPL:K FQRHATION

NEPLUK PUK SCHIST

-NFVIOISKOV FOPMATION

* NIAKGgON TUNGUF

OF CHANDLER FQRMAT ION

NIKOLAI CREEK GL ACIATIIIN

TNIKIJLA I TREENSTONE

NTKILAT FCRMATION

\begin{tabular}{|c|c|}
\hline REFEREACE & SYSTEM/SERIES \\
\hline 3 & PLEISTOCENE \\
\hline$s$ & MISSISSIPPIAN \\
\hline 10 & DEVOALAN \\
\hline $1+2$ & UPPER TRIASSIC \\
\hline 4 & JURASSIC/TRIASSIC \\
\hline $1 \mathrm{C}$ & UPPER CRETACEOUS \\
\hline 22 & JURASSIC/CRETACEOUS \\
\hline $1+2$ & UPPER TRIASSIC \\
\hline 8 & UPPEA JURASSIC \\
\hline 3 & LEMER JURASSIC \\
\hline 3 & TRIASSIC/PERMIAN \\
\hline 11 & MIDOLE DEVONIAN \\
\hline $1+2$ & CAETACEOUS \\
\hline 2 & PLETSTUCENE \\
\hline s & MTCCENE \\
\hline 3 & MISSISSIPPIAN \\
\hline 6 & UPPER DEVONIAN \\
\hline$k+2$ & LOKER CRETACEOUS \\
\hline $1+2$ & NE\$OZOIC \\
\hline 7 & PLICCENE \\
\hline $1+2$ & PRE-MISSISSIPPIAN \\
\hline 12 & OLIGOCENE/MICCENE \\
\hline 2 & UPPER CRETACEOUS \\
\hline 2 & PLE I STOCENE \\
\hline 4 & TRIASSIC \\
\hline
\end{tabular}

LOCAT IUN

SOUTHEASTERN ALASKA

AL ASKA

CANADA 6 EAST-CENTRAL ALASKA

ALASKA

CENTRAL ALASKA

SOUTHEAN ALASKA

SRITH-CENTRAL ALASKA

SOUTHEASTERN ALASKA

SOUTHWESTERN ALASKA

SOUTHHESTERN ALASKA

SOUTHEASTERN ALASKA

NURTHEASTERN ALASKA

NORTHERN ALASKa

CENTRAL SOUTHERN ALASKA

SOUTHERN AL ASKA

NORTHWESTFRN ALASKA

CENTRAL EASIERN ALASKA

CENTRAL SOUTHERN ALASKA

SOUTHEASTERN ALASKA

CENTRAL ALASKA

NUPTHERA ALASKA

SOUTHESIERA ALASKA

NORTHERN ALASKA

CENTRAL SOUTHEHN ALASKA

EASTERN ALASKA 
ALASKA

- hak hill glaciation

+HAKUSHIN VOLCANICS

- manKomen formation

HMARSHALL PASS GL ACIATION

WMARSH CREEK BRECCIA MEMBER OF T ALXEETNA FORMATION

*HASSACRE BAY FORHATIION

*hasanuska formation

4 MATANUSKA SERIES

* Melozi formation

IN SHAKTOLIK GROUP

MENTASTA ARGILLITE

MENTASTA SCHEST

DHESHIX FORMATION

MIODLE GRDUND 5HOAL MEMBER OF TYONEK FORMATION

* mint river glaciation HINT RIVER ORIFT

-MISSION CREEK SERIES

* yonte cristo dinR tTe

* moOnshine creex formation

*MOOSE CREEK MEMBER

OF TOTATLANEKA SCHIST

* moos fhora stade

*MORZHOVOI VOL CANICS

MOUNT EMMONS VOLCANICS

MOUHT FAIRPLAY GRANITE

mOUNT hague volcanics

- mount os Born glaciation

- mount susirna gLaclat ion

\section{PLEISTOCENE}

PLICCENE/PLEISTOCENE

$1+2$

3

3

12

$1+2$

$1+2$

$1+2$

3

$1+2$

5

15

3

$1+2$

$1+2$

10

$\xi$

3

3

2

3

2

2

2
PERMIAN

RECENT

LOWER JURASSIC

QUATERNARYIUPPER TERT IARY

CRETACEOUS

TERTIARY/JURASSIC

LOHER CRETACEOUS

JURASSIC/CRETACEOUS

PRE-DEVONI AN

CLIGOCENE/MIOCENE

YERTIARY

PLeistocene

FEATI IAAY / PALEOZOIC

PRE-PERMIAN

CRETACEOUS

MISSISSIPPIAN

PLeistoCEne

QUATERNARY /UPPER YERTIARY

QUATERNAAY

NCT GIVEN

QUATERNARY

PLEISTOCENE

PLEI STOCENE

\section{SOU THWESTERN ALASKA}

AL ASKA

SOUTHEASTERN ALASKA

ALASKA

SOUTHERN ALASKA

SOUTRHESTERN ALASKA

CENTRAL SOUTHERN ALASKA

ALASKA

CENTRAL HESTERN E CENTRAL aLASKA

SOUTH-CENTRAL ALASKA

SOUTHEASTEAN ALASKA

SQUTHKESIERN ALASKA

ALASKA

LLASKA

SOUTHHESTERN AL ASKA

SOUTHEASTERN ALASKA

SOUTHERN AL SKA

ALASKA

SOUTHERN ALASKA

AL ASKA

SOUTHWESTERN ALASKA

EAST-CENTRAL ALASKA

SOUTHHESTERN ALASKA

CENTRAL WESTERN aLASKa

CENTRAL SDUTHERN ALASKA 


\section{ANILKOKA CROUP}

* NINULULK FORMATION IN NANUSHUK GROUP

\&NIZINA L IKESTONE

onIZINA MOUNTAIN GORMATION

*NOAT AK SANDSTONE IN ENOICOTT GROUP NDATAK FOAMATION

* NUME GROUP

* NOME RIVER GLACIATION

* NUKa FoRmat IGN

\#NULATO FORMATION

IN SHAKTOLIK GROUP

*NUSHaGaK FORMATION

-NUTZOYIN SERIES

NUWCK FQRMATION

* OgaT ORUK FORMATION

+OKLUNE SERIES

-OKMOK VOLCANICS UKMOK ASH.

*okPikruak format ION

*orca group

* oumal ik formation

PALISADES CONGLOMERATE

* pat terson point formation

* paveloff sil istone memser

OF CHINITNA FORMATION

PAVLOF SISTER VOLLANICS

PAVLCF VOLCANICS

* PELLY GNEISS

* peratrovich formation

\begin{tabular}{|c|c|}
\hline 7 & PALECZOICAPRECAMBRI AN \\
\hline 2 & UPPER CRETACEOUS \\
\hline $1+2$ & UPPEA TRIASSIC \\
\hline 9 & JURASSIC \\
\hline 10 & MI SSISSIPPI AN/ OEVONGAN \\
\hline 11 & PRECAMBRIAN \\
\hline 2 & PLEISTOCENE \\
\hline 15 & UPPER PALEOZOIC \\
\hline $1+2$ & LCWER CRETACEOUS \\
\hline $2+2$ & MICCENESPLIOCENE \\
\hline $1+2$ & PRE-CRETACE OUS \\
\hline 2 & PLI ICENE \\
\hline 3 & JURASSIC/CRETACEOUS \\
\hline $1+2$ & TERTIARY/UPPER PALEOZOIC \\
\hline 2 & OUATERNARY \\
\hline 2 & LOWER CRETAEEOUS \\
\hline 6 & LOHER YERTIARY \\
\hline 2 & LOWER CREYACEOUS \\
\hline $1+2$ & PLEISTOCENE \\
\hline 2 & QUATERNARY \\
\hline 3 & UPPER JUAASSIC \\
\hline 2 & CUAYERNARY \\
\hline 2 & GUATERAARY \\
\hline $1+2$ & PRECAMBRIAN \\
\hline 11 & MISSISSIPPIAN \\
\hline
\end{tabular}

EASTERN ALASXA

NORTHERN ALASKA

EASTERN ILASKA

AL ASKA

NORTHERN ALASKA

NORTHKESTERN ALASKA

HEST-CENTRAL ALASKA

NORTMERN ALASKA

CENTRAL WESTERN \& CENTRAL ALASKA

SOUTHERN ALASKA

SDUTHEASTERN ALASKA

NCRTHERN ALASKA

NOR THWESTERN AL A SKA

SOUTMERN ALASKA

SOUTHEESTERN ALASKA

NOATHERN AL, SKA

LENTRAL SOUTHERN ALASKA

NORTHERN ALASKA

SOUTHWESTERN ALASKA

SOUTHWESTERN ALASKA

SOUTHERN ALASKA

SOUTHWESTERN ALASKA

SOUTHWESTERN ALASKA

CANADA E CENYRAL EASTERN ALASKA

SOUTMEASTERN ALASKA 
PETERS GLACIATION

hOINNACLE PEAK PHYLLETE.

PINNACLE (SYSTEM)

apOCHNOI VOLCANICS

*POINT HEY HEMBER OFF KATALLA FM. POINT HEY SS. MQR. DF REDWODD FM.

* pgmeror arkose member

OF NAKNEK FORMATION

ALASKA

ALASKA

SOUTHEASTERN ALASKA SOUTHEASTERN ALASKA

\section{ALASKA}

SOUTHEASTERN ALASKA

SOUTHWESTEAN ALASKA

PORCUPINE BEES

PURCUPINE GROUP

- pitrtage creek agglomerate member

$$
\text { OF TALKEETNA FORMATION }
$$

* port clarence limestone IN NOME GROUP

\#PQHT REFUGLU FORMATION

TERTIARY

* POHI CRFEK formation

*PRATT POINT MEMBER

$$
\text { OF LITTLE SITKIN DACITE }
$$

- primce creek flrmation

IN COLVIILE GROUP

FPUCKMUMA IF SCHIST

- PUfTY MSHBLR gif Katalla fORMATION PUFFY SHALE MBR. SF REDHDOD FM.

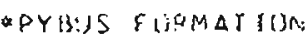
pYBUS rOL GHITE

*PYRAMIR PEAK LIMESTONE

LOHER JURASSIC

DEVCNI AN/ OROOVICI AN

UPPER DEVGNIAN

CLIGOCENE/MTCCENE

QUATERNARY

UPPER CRETACEOUS

PRECAMBRIAN

OLIGCCENE

PERMIAN

UPPER SILURIAN

CARBCNIFEROUS

PERM I AN

PRE-MISSISSIPPIAN

2 PRE-GUATERNARY
SOUTHKESTERN ALASKA

CANAOA G EASTERN ALASKA

SOUTHERN ALASKA

NORTHWESTERN ALASKA

SOUTHEASTERN ALASKA

SOUTHEASTERN ALASKA

SOUTHHESTERN ALASKA

NORTHERN ALASKA

NORTHWESTERN ALASKA

SOUTHEASTERN ALASKA

SCUTHEASTERN ALASKA

SCUTMEASTERN ALASKA

ALASKA

CENTRAL ALASKA

NORTHEKN ALASKA

ALASKA 
STRAT I CHAPMIC NAME

* rfo glacter forkation IN TUXEDN I GRQUP

RECHOOD FORHATJON

*RENOU FORMATION

*RETREAT GRQUP

*RILEY CREEK GLACIATIDN

EROAC RIVER FORHATION

* rogers creex hember

OF SCHRADER BLUFF FORMATION

RUMANZOF GRANITE

* root glacier formation

* ADLEROCHIS FORMATIDN SADL EROCHIT SANDSTONE

* sagavanirktok formation

- SAgaVANIRKTOK RIVER GLACIATION

*SAGINAH HAY FORHAIION

- sag river sanostone

ST. ELIAS SCHIST

*ST. JOSEPH islano volcanics

*S ALIGVIK GRAVEL

* SALHON LAKE glaciation

"SALMONTROUT LIMESTONE

* sanctuar formation

SANO BAY AGGLOMERATE IN SAND BAY VOL CAN ICS

SAND BAY LAVA FLOWS IA SAND BAY VOLCANICS

* 5 AND baY VOLCANICS

* SCHRADER gluff formation IN COLVILLE GROUP

*SCHRAOER GLACLATION

\section{REFERENCE SYSTEM/SERIES}

MIOOLE JURASSIC

DLIGCCENE

UPPER SILURIAN

MIDDLE OEVONIAN

PLEISTOCENE

DEVONIAN/ORDOVICIAN

10

2

3

9

11

2

2

3

11

$1+2$

11

3

2

10

10

2

2

2

2

3
UPPER CRETACEOUS

UPPER DEVONIAN

UPPER JURASSIC

TRIASSIC/PERMIAN

LOHER TERT I ARY

PLEI STOCENE

CARBONIFERUUS

UPPER TRIASSIC

NOT GIVEN

DE YONI AN

CENO2OIC

PLEISTDCENE

LOWER DEVONIAN

MI OCENE

TERT IARY

TERI I ARY

TERTIAAY

UPPER CRETACEOUS

PLEISTOCENE
LOCAT ION

SOUIHERN ALLSKA

SOUTHEASTERN ALASKA

SOUTHEASTERN ALASKA

SOUTHEASTERN ALASKA

CENTRAL SOUTHERN ALASKA

CANADA E EAST-CENTRAL ALASKA

NORT HERN ALASKA

NORTHEASTERN ALASKA

SOUTHERN ALASKA

NORTHERN ALASKA

NORTHERN ALASKA

CENTRAL NORTHERN ALASXA

SOUTHEASTERN ALASKA

NGRTHERN ALASKA

SOUTHEASTERN ALASKA

SOUTHEASTERN ALASKA

NORTHWESIERA AL ASKA

HEST-CENTRAL ALASKA

EAST-CENTRAL ALASKA

ALASKA

SOUTHWESTERN ALASKA

SOUTHWESTERN ALASKA

SOUTHWESTERN AL ASKA

NORTHEAN ALASKA 


\section{* SCHUL ze formation}

* Seaber formation IN colville group SEALEF MBR. OF SCHRAOER BLUFF IM.

\begin{tabular}{|c|c|}
\hline 10 & CRETACEOUS \\
\hline 2 & UPPER CRETACEOUS \\
\hline 3 & RECENT \\
\hline 2 & PLEISTOCENE \\
\hline \& & OLIGCCENE/MIOCENE \\
\hline 2 & UPPER CRETACEOUS \\
\hline 3 & JURASSIC/CRETACEOUS \\
\hline 3 & PLEISTOCENE \\
\hline $1+2$ & LCWER CRETACEOUS \\
\hline 3 & UPPER CRETACECUS \\
\hline $1+2$ & PALEEZOIC \\
\hline s & MISSISSIPDIAH \\
\hline $1+2$ & UPFER JURASSIC \\
\hline 2 & LDWER CRETACEOUS \\
\hline 6 & IRIASSIC \\
\hline 3 & UPPER CRETACEDUS \\
\hline 2 & LChER PERMIAN \\
\hline 3 & PLEISTOCENE \\
\hline 3 & JURASSICICRETACEOUS \\
\hline 8 & ECCENE \\
\hline 9 & CLIGCCENE \\
\hline 2 & PLEISTOCENE \\
\hline 10 & DEVOA I AN \\
\hline 3 & PLÉESTCCENE \\
\hline 11 & PERM JAN \\
\hline $1+2$ & TPIASSIC \\
\hline
\end{tabular}

\section{SOUTHERN ALASKA}

NORTHERN ALASKA

SUUTHEASTERN ALASKA

CENTRAL SOUTHEKN ALASKA

SOUTH-CENTRAL ALASKA

NORTHERN ALASKA

\section{SOUTHEASTERN ALASKA}

NORTHERN AL ASKA

CENTEAL WESTERN E CENTRAL ALASKA

NORTHERN ALASKA

SOUTHEASIERN ALASKA

CENTRAL AL ASKA

SOUTHWESTERN AL ASKA

SOUTHEASTERN ALASKA

NORJHERN ALASKA

SOUTHWESTERN ALASKA

NORTHERN ALASKA

NORTHERN ALASKA

SOUTHEASIERN ALASKA

SCUYH-C.ENIRAL ALASKA

SOUTH-CENTRAL ALASKA

SOUIHWESIERN ALASKA

NURTHERN ALASKA

SOUTHERN ALASKA

ALASKD

EASTEFN ALASKA 


\begin{tabular}{|c|c|c|}
\hline \#SKWENYNA GROUP & $1+2$ & JURASSIC \\
\hline SL ANA BASALY & 3 & TRIASSIC \\
\hline SLATE CREEK GRANODIORITE PORPHYRY & 3 & MESOLOIC \\
\hline *SLOW FORK GLACIATION & 2 & PLEI STOCENE \\
\hline $\begin{array}{c}\text { \#SNUC HARBOR SILTSIONE MEHAER } \\
\text { OF NAKNEK FORMATIBN }\end{array}$ & 3 & UPPER JURASSIC \\
\hline \#SOLOMON SCHIST & $1+2$ & PRE-CRDOVICLAN \\
\hline *S DW IK L IMESTONE & $1+2$ & OROOVICIAN \\
\hline $\begin{array}{l}\text { \#SPLIT CREEK SANOSTONE MGR KATALLA FH } \\
\text { SPLIT CREEK SHE SS MER KATALLA FM }\end{array}$ & $1+2$ & OLIGECENE \\
\hline SIANIUKDVICH FORMATION & 20 & JURASSIC/CRETACEOUS \\
\hline *STANIUKOVICH SMALE & $1+2$ & UPPER JURASSIC \\
\hline $\begin{array}{l}\text { +STAT ION CREEK FQRMATION } \\
\text { IN SKOLAI GROUP }\end{array}$ & 11 & PERMIAN \\
\hline *SIEP CONGLOMERATE & 10 & PERAIAN \\
\hline \#STEPHENS PASSAGE GROUP & 3 & JURASSICICRETACEOUS \\
\hline $\begin{array}{l}\text { STEPOVAK FORHATION } \\
\text { IN GEAVER BAY GROUP }\end{array}$ & 3 & LOHER TERTI ARY \\
\hline STEPOVAK SERIES & $1+2$ & EOCENE \\
\hline $\begin{array}{l}\text { STERL ING FORMATION } \\
\text { IN KENAI GROUP }\end{array}$ & 16 & TERTIARY \\
\hline *STILLHATER FORMATION & $1+2$ & EOCENE \\
\hline \#STRELNA FORHATION & $1+2$ & MISSISSIPPIAN \\
\hline $\begin{array}{l}\text {-5TURGEON RIVER GLACIATIUN } \\
\text { STURGEON RIVER DRIFT }\end{array}$ & 11 & PLEISTOCENE \\
\hline 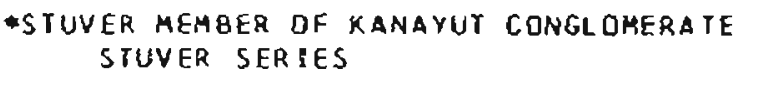 & $1+2$ & UPPER DEVONIAN \\
\hline *SUMMIT LAKE GLACIATION & 3 & PLEISTOCENE \\
\hline +SUNRISE SERIFS OR GROUP & $1+2$ & MESOZOICIPALEOZOIC \\
\hline *SUNTRANA FORHATION & 10 & MICCENE \\
\hline *SUSITNA SLATE & $1+2$ & JURASSIC/CREIACEOUS \\
\hline SUSLOTA DIORITE & 3 & TERTIARY \\
\hline
\end{tabular}

SOUTHERN ALASKA

SOUTH-CENYRAL ALASKA

ALASKA

SOUTH-CENTRAL ALASKA

SOUTHERN ALASKA

NDRTHKESTERN ALASKA

NORTHWESTERN ALASXA

SOUTHEASTERN ALASKA

SQUTHKESTERN ALASKA

SOUTKHESTERN ALASKA

ALASKA

EAST-CENTRAL ALASKA

SOUTHEASTERN AL SKKA

SOUTHERN ALASKA

SOUTHWESTERN ALASKA

ALASKA

SOUTHEASTERN ALASKA

SOUTREASIERN ALASKA

ALASKA

NORYHERN ALASKA

ALASKA

SOUTHERN ALASKA

ALASKA

SOUTHERN ALASKA

SOUTHECENTRAL ALASKA 
* Susleta limestone

SWAN LAKE GLACIATION

- SYMDNOS FORMATION

+T aChaYna series

* Y ACHILNI FORMAYION

* AHKSNDIT LIMESTONE

+ TAHK ANOIT SERIES

- takotana fornayion

- t alkeerina formation

m TANAK VOLCANICS

+t anana schist

* t anya STADF

TANYA SUBSTAGE

TYT AL INA GROUP

- tatina group

*telavirak formation

IERRA COTIA SEPIES

* etflina volcanics

*TEXAS CKEEK GRANDOIDR ITE

* y haNE vitlcanir, group

THETIS CROUP

GT IDAL FORMATIIIN

* T ivakaha schist IN KIGLUAIX gROUP

* I GLUKPUK FORMATIUN

- indir group

* TlFVAK BASAL $T$

TUG S $\triangle K$ GRAVELS

TOK SANCSTONF

\begin{tabular}{|c|c|}
\hline $1+2$ & PENNSYLVANIAN \\
\hline 2 & PLEISTOCENE \\
\hline 2 & LOHER CRETACEOUS \\
\hline $1+2$ & HIDDLE DEVONIAN \\
\hline$\cdot 3$ & UPFER TERTIARY \\
\hline 12 & LQWER PERMIAN \\
\hline $1+2$ & PERMIAN/PENASYLVANIAN \\
\hline $1+2$ & MIDOLE DEVDNIAN \\
\hline 8 & LOWER JURASSIC \\
\hline 2 & QUATERNARY \\
\hline $1+2$ & PRECAMBAIAN \\
\hline 3 & RECENT \\
\hline $1+2$ & PRE-SILURIAN \\
\hline $1+2$ & SILURIAN/ OROOVICIAN \\
\hline 3 & JURASSIC/CRETACEOUS \\
\hline $1+2$ & OE VOA I AN/OROUVICIAN \\
\hline $1+2$ & CARBCHIFEROUS \\
\hline $1+2$ & JURASSICICRETACECUS \\
\hline $1+2$ & JURASSIC \\
\hline $1+2$ & UPPER CRETACEOUS \\
\hline 3 & UPPER SLLURIAN \\
\hline $1+2$ & PRE-MISSISSIPPIAN \\
\hline 2 & LIPPER JURASSIC \\
\hline $\mathrm{e}$ & PRECAMBRIAN \\
\hline$\varsigma$ & CENDZOIC \\
\hline $1+2$ & PLEISTOCENE \\
\hline 2 & TERTIARY \\
\hline
\end{tabular}

CENTRAL EASTERN ALASKA

CENTRAL SUUTHERN ALASKA

SOUTHEASTERN ALASKA

SOUTHERN ALASKA

ALASKA

EAST-CENTRAL ALASKA

EASTERN AL ASKA

SOUTHERN ALASKA

CENTRAL SOUTHERN ALASKa

SDUTHWESTERN ALASKA

EASIERN AL ASKA

SOUTHERN ALASKA

NORTHEASTERN AL ASKA

CENTRAL SOUTHERN ALASKA

NORTHWESTERN ALASKA

SOUTHERN ALASKA

SOUTHEASTERN ALASKA

SOUTHEASTERN ALASKA

SOUTHEASTERN ALASKA

NORYHWESTERN ALASKA

SOUTHEASTERN AL ASKA

NURTHWESTERN ALASKA

NORTHERN AL ASKA

EAST-CENTRAL AL ASXA

SOUTHEASTERN ALASKA

SOUTHEESTERN ALASKA

EASTERN AL ASKA 
*tokUn furmarron

m OLLOVANA L IHESTUNG

TOLSTOT FURMATION IN BEAVER GAY GROUP

*TONNIE SILTSTONE MEMBER, ChINTINA FM. TONNIE SS OR SLTST MBR, TUXEONI FM

*tunzona group

*T apAGORUK Formation TOPAGORUK MEMBER OF UMIAT FM.

* tgps y formatinn

*T TRDRILLO FORMAT ION

* ToROK FORMAT ION

*TOTATLANIKA SCHIST

+TOTSEN SERIES OR GRQUP

-TRELONELL SLATE OR FORMATION

* ISADAKa Formation

*TUGIDAK FORMATION

* YUKTU FORMATION IN NANUSHUK GROLP TUKTU MEMBER OF UMIAT FORMATION

- tulik basalt

TTULUGA MEMAER

OF SCHRADFR BLUFF FORMATION

* JULUVAK tONGUE

OF PRINCE CAEEK FORMATION

* TUNAEL STAOE TUNNEL DRIFT

ATUPIK FORMATION

IN I IS QURNE GROUP

* TUSTUMENa STADE TUSTUHENA DR IFT

*TUXEIDNI GROLP

TUEEONI SANDSTONE OR FORMAYYOA

THELVEMILE BEOS

* IHIS T CREEK SILTSTONE IN TUXEDNI GROUP

\begin{tabular}{|c|c|}
\hline $1+2$ & EOCEAE/OLIGOCENE \\
\hline 7 & DEVDNI AN/SILURIAN \\
\hline 3 & PALECCENE/EDCENE \\
\hline 2 & UPPER JURASSIC \\
\hline $1+2$ & PALECZOIC \\
\hline 2 & LOMER CRETACEOUS \\
\hline 8 & OLIGCCENE/MIOCENE \\
\hline $1+2$ & MIDOLE JURASSTC \\
\hline 2 & LOWER CRETACEOUS \\
\hline$\varsigma$ & MISSISSIPPIAN \\
\hline $1+2$ & LOWER PALEOLOIC \\
\hline $1+2$ & JURASSIC/CRETACEOUS \\
\hline 10 & MIOCENE \\
\hline s & PLIOCENE \\
\hline 2 & LOKER CRETACEOUS \\
\hline 2 & CENOZOIC \\
\hline 2 & UPPER CRETACEOUS \\
\hline 2 & UPPEA CRETACEOUS \\
\hline 3 & RECENT \\
\hline 3 & UPPER MISSISSIPPIAN \\
\hline 3 & RECENT \\
\hline 4 & JURASSIC \\
\hline$I+2$ & TERT IARY \\
\hline 3 & MIODLE JURASSIC \\
\hline
\end{tabular}

SOUTHEASTERN ALASKA NORTHEASTERN ALASKA SOUTHWESTERN ALASKA

CENTRAL SOUTHERN ALASKA

CENTRAL SOUTHERN ALASKA

NORTHERN AL ASKA

SOUTHERN ALASKA

CENTRAL SOUTHERN ALASKA

NORTHERN ALASKA

CENTRAL ALASKA

NORTHERN ALASKA

SOUTHEASTERN ALASKA

SOUTH-CENTRAL ALASKA

SOUTH-CENTRAL ALASKA

NORTHERN ALASKA

SOUTHKESTERN ALASKA

NORTHERN ALASKA

NORTHERN ALASKA

ALASKA

NORTHERN ALASKA

ALASKA

CENTRAL SOUTHERN ALASKA

SOUTHWESTERN ALASKA

SOUTHERN ALASKA 


\section{REFEREACE}

SYSTEM/SERIES

*TYONER FURMATION

IN KFNAI GRCUP

*uL ANFAK CRFEK gLACIATION

UMIAY bENTINITE DF SEABEE FORMATION

+UMIAT FIIRHATION

IN NANUSHUK GRQUP

*unal aska formas ion

UNALUX GL ACIATILN

UNALUK ORIFI

*unca cbnglomerate

* UNGALIK CONGL OMERate

* utukgk formatigin

IN LIS BUPNE GROUP

*UYAK FORMATION

*VALDEZ GRQUP

SVALLENAK FORMAIION

VAN HGRN FORHATION

* vega bay formation

VILCANO GAY HASALT FLOH

IN MOUNS haGUE VGLCANICS

*WACHSMUTH LIMESTONF.

IN L ISTURAT GOOUP

WACLFIGH LIMKSTONF

EHATON LIMESTIINI

IN LISBUFAE CPOOLP

inALES GRTUP

NWALKER LARF GLACIAT ION

WWATFRBUITT EASALT

*hajerfall greenstone

*WELLER TLLACIATION

WELLESLFY FILRMATION

\begin{tabular}{|c|c|}
\hline 16 & TERTIARY \\
\hline 3 & RECENT \\
\hline 3 & CRETACEUUS \\
\hline 2 & CRET ACEOUS \\
\hline 3 & TERYIARY \\
\hline 3 & PLEISTOCENE \\
\hline 8 & MICCENE \\
\hline $1+2$ & LEWER CRETACEOUS \\
\hline 3 & LOHER MISSISSIPQIAN \\
\hline 5 & TRIASSIC \\
\hline 7 & JURASS ICICRETACEOUS \\
\hline $1+2$ & MIDOLE OEVONIAN \\
\hline 3 & PLEISTGCENE \\
\hline 3 & TERT IARY \\
\hline 2 & CUATERAARY \\
\hline 11 & MISSISSIPPIAN \\
\hline 11 & DEVCASAN \\
\hline 12 & PERMIANI PENNSY LVANIAN \\
\hline $1+2$ & PRE-NISSISSIPPISN \\
\hline 3 & PLEISTOCENE \\
\hline $1+2$ & MICCENE/PLIOCENE \\
\hline 3 & TRIASSIC \\
\hline 3 & PLEISYOCENE \\
\hline $1+2$ & MISSISSIPPIAN \\
\hline
\end{tabular}

LOCATION

SQUTHERN ALASKA

NORTHWESTERN ALASKA

NORTHERN ALASKA

NORTHERN ALASKA

AL. ASKA

SOUTHWESTERN ALASKA

SOUTHHESTERN AL SKA

CENTRAL WESTERN E CENTRAL ALASKA

NORTHERN ALASKA

SCUTH-CENTRAL ALASKA

CENTRAL SOUTHERN ALASKA

SOUTHEASTERN ALASKA

SOUTHEASIERN ALASKA

SOUTHEPN ALASKA

SCUTHWESTERN ALASKA

NURTHERN ALASKA

SCUTHEASTERN ALASKA

NORYHEHN AEASKA

SOUTHEASTERN ALASKA

NORTHWESTERN ALASKA

SOUTHHESTERN ALASKA

SOUTHEASIERN ALASKA

AL ASKA

EASTERN ALASKA 
*hest cove memger

DF LIYTLE SITKIN DACITE

WEST FORELAND FORMATIOA

IN KENAI GROUP

* HEST FORK FORHATIDN

WEST FORK GRANODIORITE

*WHIIESTRIPE MARBLE

WILL IAM HENRY BAY HARELE

* Hilliwan cove formation

*h illoughar l. tmestane

*WISHBONE FORMATION

WOLVERINE COMPLEX

*honger lake glaciation

*hocochoppfa volcanics

* hurt hington Gl ac iat ION

*WR ANGELL LAVA

-yakajaGa fORMation

-yakutat group

+YENTNA BEDS

*YORK GLACIATION YORK DRIFT

YUKON GROUP

YUKON SILTS

- ZETO POINT BASAL PORPHYRY
SOUTHWESTERN ALASKA

ALASKA

NORTHERN ALASKA

ALASKA

SOUTHEASTERN ALASKA

SOUTHEASTERN ALASKA

SOUTHHESTERN ALASKA

SOUTHEASTERN ALA SKA

SOUTH-CENTRAL ALASKA

SOUTH-CENTRAL ALASKA

SOUTH-CENTRAL ALASKA

NORTHEASTERN ALASKA

ALASKA

SOUTHERN ALASKA

SOUTHERN ALASKA

SOUTHERN ALASKA

SQUTHERN ALASKA

ALASKA

NORTMEASTERN ALASKA E CANADA

NORTHEASTERN ALASKA

SOUTHWESTERN AL ASKA 


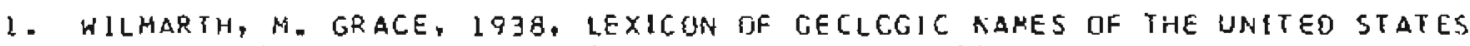
IINCLUDING ALASKA), W.S.G.S. BULL. 856. $2396 \mathrm{pP.}$

2. KERGHFR, GRACE C., ANO OTHERS, LYGE. LEIICCA OF GEGLOGIC NAMES OF THE UNITFD STATFS FOR 1930-196C, U.S.G.5. BLLL. 1200, 434L PP.

3. KEROMER, CPACE C., 297C, LEXICUN OF CEClOGlC NAHES CF the UNITEQ SIATES FOR 1961-1967, U.5.G.5. QULL. 1350, E46 PP.

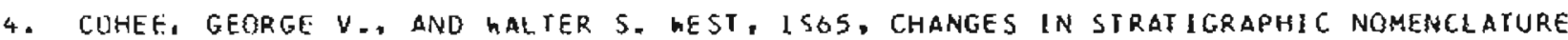
BY THE U. 5. GEOLOGICAL SURVEY, 1563, L.S.G.S. BULL. 1194-A. PP. Al-A2L.

5. COHEE, GEORGE $V$. AND hALTEA S. HEST, 1965 , CHANGES IN STRATIGRAPHIC NCMENCLATURE BY THE U. S. GEOLOGICAL SURVEY, 1564. L.S.G.S. BULL- 1224-A, PP. AL-AZ2.

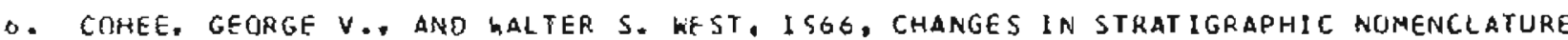
8Y THE U. S. GEOLOGICAL SURVEY, 1565, U.S.G.S. BULL. 1244-A, PP. AL-A22.

7. CJHEE, GEORGE V.. HALTER S. HEST, AND LCRNA C. WLLKIE, 1967, CHANGES IN STRATIGRAPHIC NDMENCLATURE BY THE U. S. GEOLOGIGAL SLRVEY, 1966. U.S.6.S. BULL. 1254-A, PP. AI-AIT.

H. COHFE, GEORGE V., ROBERT G. BATES, and WLNa B. hright, 1969. CHANGES IN STRATIGRAPHIC NOMENCLATURE DY THE U. 5. GeOLOGICAL SLPVEY, 1967, U.5.G.5. BULl. 1274-A, PP. AL-AZI.

7. COHEF, GEORGE V., RLBERT G. BATES, ANO VILNA B. HRIGHT, 1970, CHANGES IN STRATIGKAPHIC NOMENGLATURE BY THE U. S. GEOLOGLCAL SLRVEY, 1968, U.S.G.S. BULL. 1294-A, PP. AL-A20.

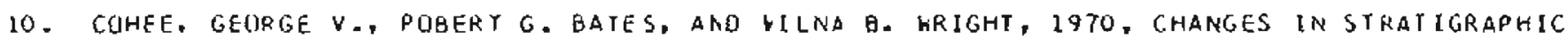
NOMENCLATURE BY THE U. 5. GEOLOGICAL SURVEY, 1969, U.S.G.5. BULL. 1324-A, PP. Al-A 15.

11. COHEE, GEORGE V., GUBERT G. BATES, ARD bILNA 8. hRIGHT, 197L, CHANGES IN SIRAIIGRAPHIC NCMENCLATURE BY THE U. S. GFOLUGICAL SUFVEY, 1970, U.S.G.S. BULL. 1354 A, PP. Al-A29.

12. COHFE, GFQRGE V.. AND MILNA B. WPIGHT, IS72, CMANGES IA STRATIGRGPHIC MGMENCLATURE BY IHF U. S. GEULOGICAL SURVEY, LS7L, U.S.G-S. BULL. 1372-A, PP.ALAZB.

13. KELI.FR, SAMUEL, ROBERT H. MCIKRIS, AND RCHERT L. OETTERMAN, 1961, GELLCGY OFF THE SHAV SOVIK AND SAGAVANIRKTOK RIVERS KEGIEN, DLASKA, U.S.G.S. PRUFESS IUNAL PAPER 3O3-O, PP. $167-222$.

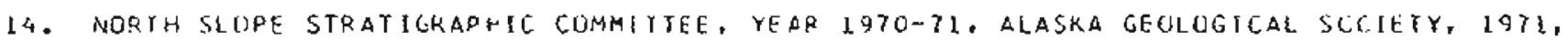
WESY TO EAST STRATIGRAPHIC CORRELATICN SECIICA. POINT BARROH TO IGNEK VALLEY, ARCTLC NORTH SLQPE, ALASKA.

15. STRATIGRAPHIC CUMMHTYEE, YFAR 1968-1569, ALASKA GEELOGICAL SUC!FTY, 146\%, CCUK INLET BASIN STRATIGRAPHY STUDY, 1969.

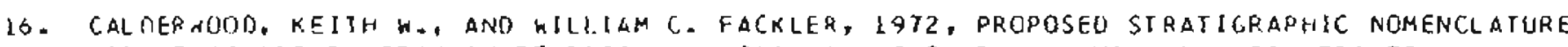
FOK KENAI GRDUP, COJK INLE I BASIN, ALASKA, A.A.P.G. BULL. VOL. 56, PP. 739-754.

17. MULL, C. G., $\triangle N E$ M. O. HANGUS, 1972, ITKILYYRIAK FERMATION: AEH MISSISSIPPIAN FORHATION OF ENDICOTY GROUP, ARCTIC SLOPE OF ALASKA, A.A.P.G. 8ULL., VOL. 56. PP. $1364-1369$.

1月. MEFTH SLOPE STRATIGRAPHIC CUMHITEEF, ALASKa GEQLDGICAL SOCIETY, 1970, IHE SAG RIVEK SANDSTRIEE AND KUPARUK RIVER SANDS, THO INPCRTANT SUBSURFACE UAITS IN THE PRUOHOE BAY FIELO, IN PROCEEDINGS OF TES GFGLCCICAL SEFINAR CN THE NORTH SLOPE OF ALASKA, WUBI ISHF UY PACIFIC SECTION A.A.P.G.. FP. PI-P3. 
19. TAILLEUR, 1. L.. B. L. MAMET, AND J. I. QUYRB, JR., 1973, REVISEO AGE ANO STRUCTURAL INTERPRET ATIONS OF NUKA FORMATION AT NLKA RIDGE, NORTHWESTERN ALASKA, A.A.P.G. BULL., VOL. 57, PP. 1348-13E2.

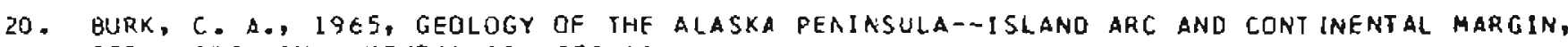
GEOL, SOC. AM., MEMOTR 99, 250 PP.

21. CLARK, SANDRA H. 8.. 1S72, THE KOLVERIRE CCMPLEX, \& NEWLY CISCCVEREO LAYERED ULTRAMAFIC BOOY IN THE WFSTERN CHUGACH HGURTAINS, ALASKA, U.S.G.S. DPEN FILE REPORT, IO PP.

22. CLARK, SANORA H. 8., 1973, THE MCHUGH CCAPLEX CF SCUTHMCENTRAL ALASKA, U.S.G.S. BULC. 1372-D, PP. DI-D11. 